\title{
A dual-PMT optical module (D-Egg) for IceCube-Gen2
}

\section{The IceCube-Gen2 Collaboration ${ }^{\dagger}$, H. Ijiri ${ }^{1}$, L. Lu ${ }^{1}$}

${ }^{\dagger}$ http://icecube.wisc.edu/collaboration/authors/icrc15_gen2

${ }^{1}$ Department of Physics, Chiba University, Chiba 263-8522, Japan

E-mail: lu.lu@icecube.wisc.edu

The upgrade of the IceCube neutrino observatory (IceCube-Gen2) enhances the detection capability of neutrinos with a few hundred $\mathrm{TeV}$ energies or greater by the increased instrumented volume in the glacier ice. Enhancement of the optical sensor performance in detecting ultraviolet photons can be a key factor for IceCube-Gen2 to achieve a higher sensitivity as more Cherenkov photons are expected in the short wavelengths. We have developed an optical module housing two $8^{\prime \prime}$ photomultiplier tubes (PMTs) in an UV transparent, oval-shaped glass housing. The two high-QE PMTs are installed in a way facing both up and down so that the resultant angular acceptance is more uniform. This uniformity of optical acceptance further improves the detection of downward-going events and background veto efficiency compared to the current IceCube optical sensors. In addition, the improvements on UV transmittance of the glass housing and the inner gel lead to an improvement of the photon detection efficiency by a factor of four at wavelengths shorter than $340 \mathrm{~nm}$.

Here, the initial performance of the first prototype module of D-Egg is reported. We also present simulation studies of the IceCube-Gen2 performance with the new dual-PMT modules.

Corresponding authors: L. Lu*1, A. Ishihara ${ }^{1}, \mathrm{~S}$. Yoshida ${ }^{1}$

${ }^{1}$ Department of Physics, Chiba University, Chiba 263-8522, Japan

The 34th International Cosmic Ray Conference,

30 July- 6 August, 2015

The Hague, The Netherlands

${ }^{*}$ Speaker. 
The high-energy extension of the IceCube experiment aims at improving the sensitivity for the detection of neutrinos with few hundreds of $\mathrm{TeVs}$ and energies beyond. To achieve this, a larger instrumented volume is needed and the spacing between strings will be increased [1]. To improve the efficiency for detecting Cherenkov photons, one can use new types of glass-housings that are more UV-transparent. In addition, the direction information of photons might help with vetoing atmospheric muon backgrounds. The design of the new detector, D-Egg (Dual optical sensors in an Ellipsoid Glass for Gen2), has been optimised to increase the detection efficiency for UV photons and angular acceptance at a minimum additional cost. The advances of D-Egg make it also an excellent candidate to be the light sensors of the low-energy upgrade, PINGU [2], which focuses on precise measurements of low-energy neutrinos in the ice.

The concept of the D-Egg in more details is introduced in Section 1. In Section 2, the new optical module is simulated using GEANT4 [3] and the angular sensitivity is compared to the current IceCube. Measurements of the properties of D-Egg in the lab are also discussed. Next in Section 3 , the gain of performance when using D-Egg is illustrated by simulating muons in the ice.

\section{The concept of D-Egg}

The current Digital Optical Module (DOM) in IceCube carries one 10" PMT (Hamamatsu R7081-2) looking down to the ice [4]. It has been proven to be reliable and with satisfactory performance. The D-Egg carries two 8" PMTs (Hamamatsu R5912-100) with one looking up and one looking down as shown in Figure 1.

One unique feature of D-Egg is that the depth of the glass varies along the latitude, being thicker at its equator and thinning at the pole. The design of the thickness were carefully optimised for both requirements from optical performance and mechanical strength, which are usually contradicted to each other. The mechanical tolerance has been verified in the pressure vessel with $\mathrm{P}=70 \mathrm{MPa}$, which is higher than the greatest pressure observed so far during the freeze-in process of the current IceCube. The diameter of D-Egg is $300 \mathrm{~mm}$, which is $30.2 \mathrm{~mm}$ smaller than the current IceCube DOM and will reduce the drilling cost. The electronics and digital readout are modified based on the designs of the Gen2 baseline DOM [5].

Another important characteristic of D-Egg is the enhancement of the efficiency for detecting UV photons. The emission spectrum of the Cherenkov radiation can be described by $1 / \lambda^{2}$, therefore it is key for detectors to have high acceptance in the UV range. The absorption length of photons of different wavelengths varies with depth due to

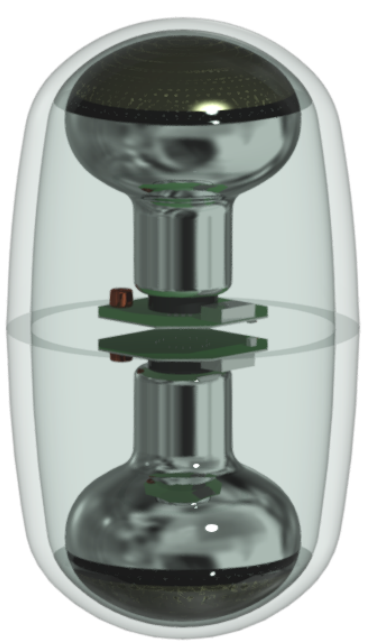
different optical properties of the ice layers. In the deepest clean ice, the absorption length of $380 \mathrm{~nm}$ photons is $\sim 200 \mathrm{~m}$ while it is only $\sim 40 \mathrm{~m}$ in the dust 
layer. At the depth of $\sim 400 \mathrm{~m}$ above the dust layer, the absorption length of photons of $\lambda \sim 350 \mathrm{~nm}$ is $135 \mathrm{~m}$, which is only $15 \mathrm{~m}$ shorter than photons of $\lambda \sim 400 \mathrm{~nm}$.

For the current IceCube DOM, the glass used to house the PMT absorbs $40 \%$ of photons with $350 \mathrm{~nm}$. An improved type of glass has been chosen for housing D-Egg. By removing the iron contamination in the glass and reducing the thickness by $0.27 \mathrm{~cm}$, nearly all photons of $350 \mathrm{~nm}$ pass through the glass vertically at the pole. A new type of gel has been used and the thickness remains the same with the current DOM. The transmittance is shown in Figure 2(a).

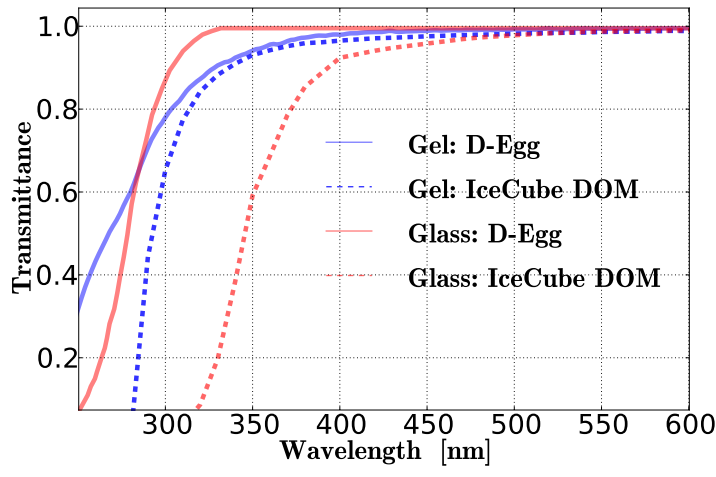

(a)

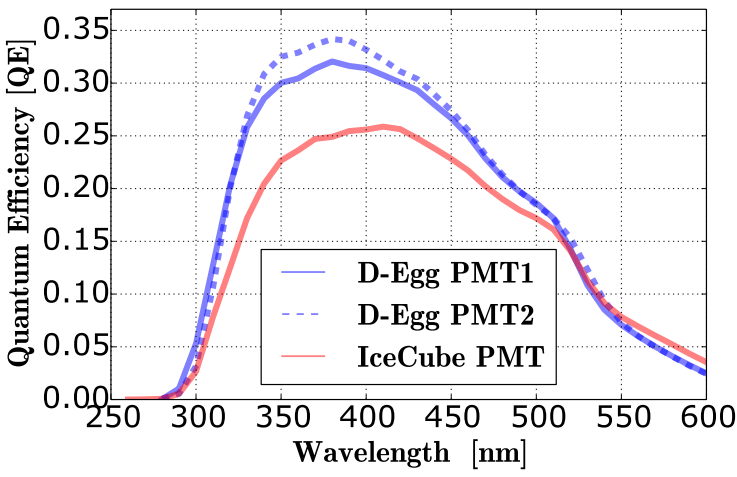

(b)

Figure 2: (a) Transmittance of glass and gel for photons with different wavelengths for the current IceCube DOM and D-Egg. (b) The comparison of Quantum Efficiency (QE) of the current IceCube 10" and the DEgg 8" PMT. The curves are from Hamamatsu measurements [6] without corrections for collection efficiency or charge response. It has also been proposed to use the high QE PMTs which are currently taking data in the DeepCore [7] for the next generation of IceCube. The DeepCore PMTs have similar QE with the 8" PMTs in D-Egg.

The quantum efficiencies of the 8 " PMTs are higher especially at the UV range, as shown in Figure 2(b). At wavelength $\lambda=380 \mathrm{~nm}, \mathrm{QE}$ is improved from $25 \%$ to $\sim 33 \%$. The combined effect of using the new glass and PMT significantly enhance the detection of UV photons. In the next section, simulations of the photon propagation in the glass, gel and PMT are performed and the quantum efficiency of the detector is compared with measurements made in the lab.

\section{Detector simulations and lab measurements}

The geometry and optical properties of D-Egg have been implemented in GEANT4. Photons of wavelengths $300 \mathrm{~nm}$ to $650 \mathrm{~nm}$ were simulated in circular beams. The beams are rotated from the bottom to the top of the DOM and have a diameter of $0.534 \mathrm{~m}$, which is the same as the height of D-Egg. The characteristics of the new PMTs measured in the lab, such as the charge response and the uniformity of the collection efficiency are included in the PMT simulations.

The effective area for all wavelengths and zenith angles for the IceCube DOM and D-Egg is shown in Figure 3. It is calculated as the ratio of the number of photoelectrons generated by the PMT over the number of incident photons. It is clear that the IceCube DOM is mostly sensitive to photons from below the DOM with zenith angle $\theta \geq 90^{\circ}$. On the other hand D-Egg has nearly 
isotropic angular acceptance. At $340 \mathrm{~nm}$, the effective area of D-Egg integrated over all angles is $\sim 4$ times the effective area of the spherical DOM.

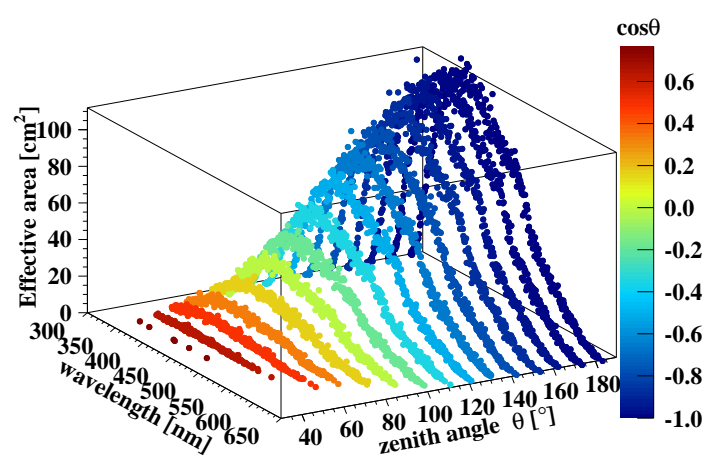

(a)

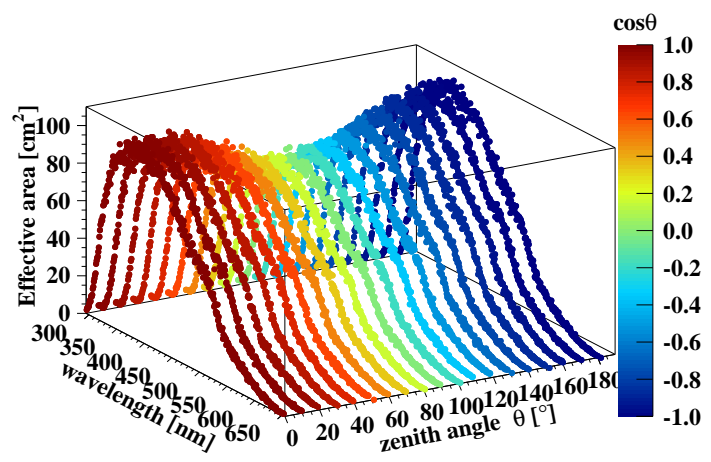

(b)

Figure 3: The effective area of the current IceCube spherical DOM (a) and D-Egg (b). The optical properties of glass and gel have been implemented in GEANT4 simulations. The PMT simulations include the charge response correction, quantum and collection efficiencies. Each point scales with the probability of photon of certain wavelength and zenith angle being converted to photoelectrons.

It is important to verify the simulations using measurements in the lab. Figure 4 shows the setup in the lab for measuring the quantum efficiency of D-Egg in a freezer. Five LEDs $(340,365,470,520,572 \mathrm{~nm})$ are used as light sources. A fraction of the photons are split from the beam and goes to a small (monitor) PMT that has well-known gain. The ratio of reflection over transmission for the beam splitter is measured for each run. One could estimate the number of photons reaching the big PMT by scaling the number of photons observed via the small PMT.

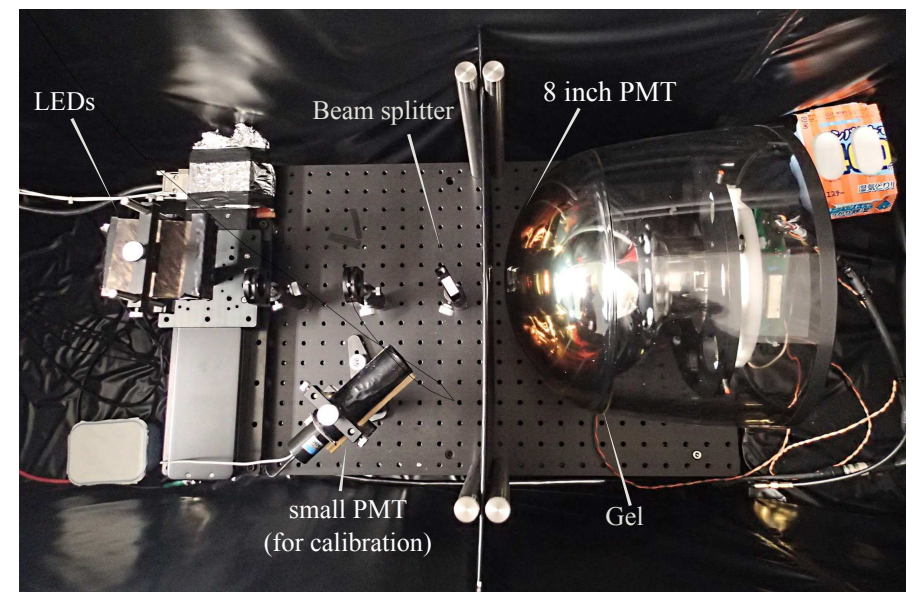

Figure 4: Lab setup for measuring quantum efficiency of D-Egg (with glass and gel) in the freezer. There are five LEDs contained in the wooden box on the left. The position of the LEDs are shifted using an automatic base so that the measurements will not be disturbed when the freezer is closed.

The quantum efficiency of the bare PMT was measured first and is about $88 \%$ of the Hamamatsu result due to the absence of the charge response and reflectivity correction. With taking into 
account the glass and gel, D-Egg has a wavelength acceptance at $350 \mathrm{~nm}$ of nearly $26 \%$, which is $13 \%$ higher than that of the current IceCube DOM. A comparison between lab measurements and simulations for D-Egg is shown in Figure 5.

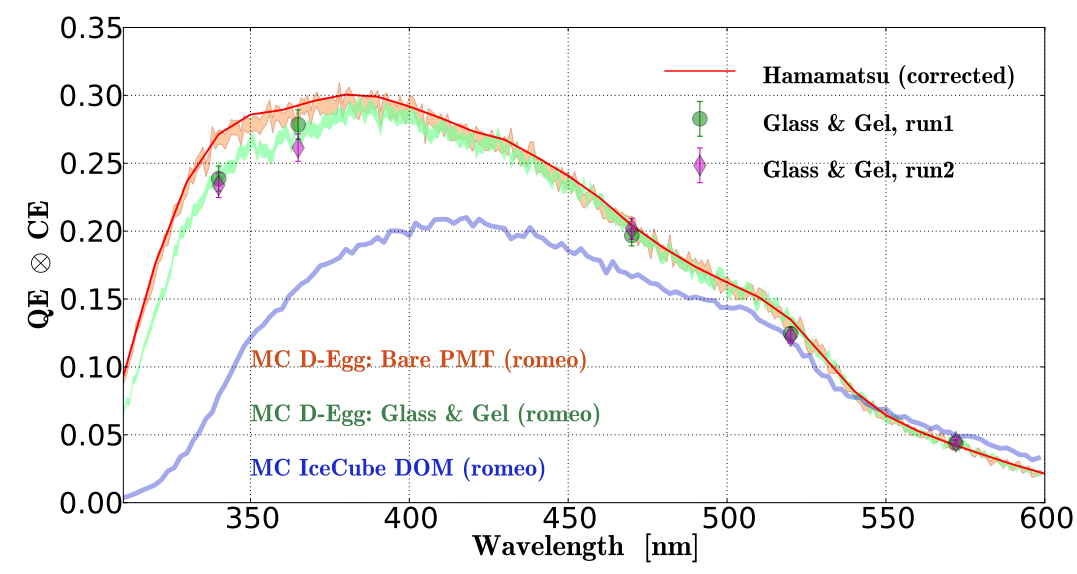

Figure 5: Comparisons of quantum efficiency measured in lab and simulations. The simulations are based on GEANT4 and romeo. Bare-PMT simulation is compared with Hamamatsu measurements which have been corrected for charge response and reflectivity. The blue curve shows the simulated efficiency when photons hit the centre of the photocathode of an IceCube DOM.

It has been demonstrated that D-Egg has great advantages on angular sensitivity and UV photon-detections. In the next section, the importance and improvement of using D-Egg is shown by looking at physics events using muon simulations.

\section{The physics case of using D-Egg}

Atmospheric muons are a tricky background for neutrino searches and it is vital to identify them. The improvements of angular and UV photon sensitivity using D-Egg ultimately provide additional information on physics processes in the ice, which are useful for rejecting muon backgrounds and reconstructing the event geometry. In this section, first an example of muon event is shown to illustrate the importance of having the up-looking PMT, followed by a discussion of how the veto could be improved using D-Egg.

\subsection{An example of a muon event}

A down-going muon with zenith angle $50^{\circ}$ is shown in Figure 6(a). The Monte Carlo energy of the muon is $4 \mathrm{TeV}$ and $540 \mathrm{GeV}$ is lost through bremsstrahlung. The energy loss was very close to the DOM $(\sim 20 \mathrm{~m})$ and photons experience few scatterings. The emitted photons are in the forward direction of the track and a large fraction hit directly on top of the DOM 60, which is located at the bottom level on string 41 . The muon only develops at a 'corner' of the IceCube detector and partly contained.

The larger the distance, the more scatterings the photons go through so that the zenith angles become more homogeneous. Due to the short distance, the average number of scatterings for photons arriving on DOM 59 and 60 is about 5, which means these photons nearly do not change 


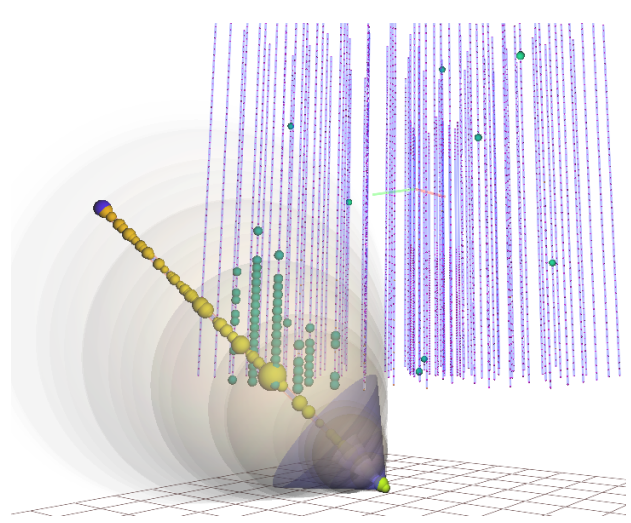

(a)

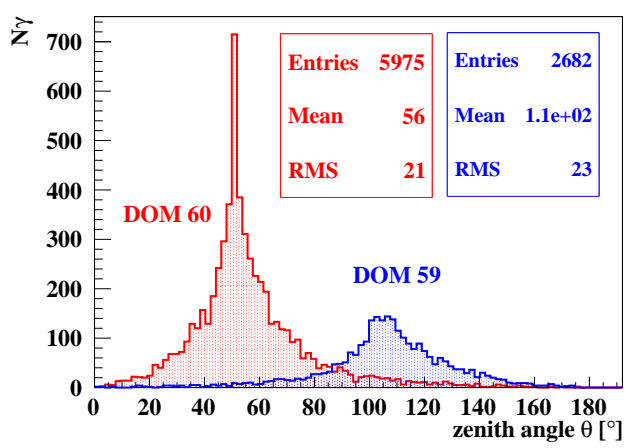

(b)

Figure 6: (a) The example event that has DOMs close to an energy loss of $540 \mathrm{GeV}$ through bremsstrahlung. DOM 59 and 60 are on string 41 with DOM 59 above DOM 60. Photons undergo $\sim 5$ scatterings and a huge fraction of them arrive on the top of DOM 60 (zenith angle smaller than $90^{\circ}$ ). (b) Distribution of zenith angle for photons when arriving on DOM 59 and 60. The angles are results of post-scatterings.

the direction of propagations. The distribution of zenith angle for each photon arriving on DOM 59 and 60 is shown in Figure 6(b). As expected DOM 60 receives majority of photons from the top and DOM 59 receives most photons from the bottom of the detector.

Photons hitting the top of the DOMs with $\theta=50^{\circ}$ have low probability of being detected by the IceCube DOM (Figure 3(a)). The number of photons which hit the DOMs as a function of the in-ice depth is shown in Figure 7 (left). The simulation was done by tracing each Cherenkov photon using GEANT4. Once the photons arrive at the DOMs, they are passed on to the detector simulation of IceCube DOM and D-Egg in parallel. The distribution of photoelectrons is shown in Figure 7 (right) for both D-Egg and IceCube DOM.
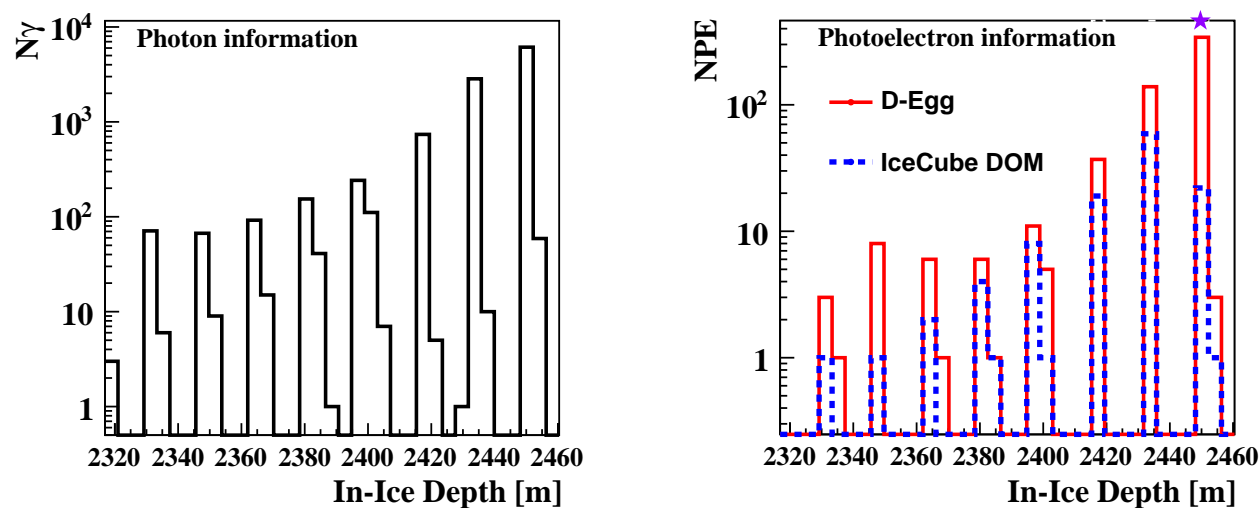

Figure 7: Left: The photon profile as the muon travels through the ice. The event happened close to the bottom layer of the ice. Only photons that arrive on the DOMs are counted. Right: The photoelectron profile as detected by D-Egg and IceCube DOM. 'NPE' stands for number of photoelectrons. The gaps between the depths are due to the distance intervals between DOMs on the strings.

The number of photoelectrons generated in DOM 60 is highly suppressed in the case of the IceCube DOM. The depth of DOM 60 is marked as a star. It is evident from Figure 7 that the photon 
profile of this event can only be reproduced by using the photoelectron information provided by D-Egg. The photoelectron map from the IceCube DOM is inaccurate, which could have serious impacts on the interpretation of the zenith angle and the energy of the event. More studies regarding this issue have been planed in the future with dedicated simulation samples.

\subsection{Potential for improving self-veto}

One of the popular methods to reject muon backgrounds is to count the number of photoelectrons on the outer layer of DOMs. In such way neutrinos that have vertex inside the detector are selected. The background muons come from the atmosphere and in principle generate Cherenkov photons hitting the top of the DOMs, which is not ideal for the down-looking PMTs. However, photons are scattered in the ice and eventually the angular distributions become more isotropic unless the DOMs are close to the interaction points and direct light reaches the DOM. To understand how D-Egg could help for self-veto [8], single-muon events are simulated with energies from $100 \mathrm{GeV}$ to $10 \mathrm{TeV}$.

Typically for the current IceCube the ice in the holes has different optical properties compared with the ice between the strings [9]. The scattering length is much shorter than that in the bulk ice due to the drilling process. However, for the Gen2 upgrade more precautions [1] will be made and one expects much clearer ice in the holes. Therefore for simulations included in this study, the hole ice is considered as the same with the bulk ice. Vertical muons with $\theta<40^{\circ}$ are selected. Figure 8 shows the distribution of zenith angles of the photons that hit the DOMs for all the selected 1052 events.

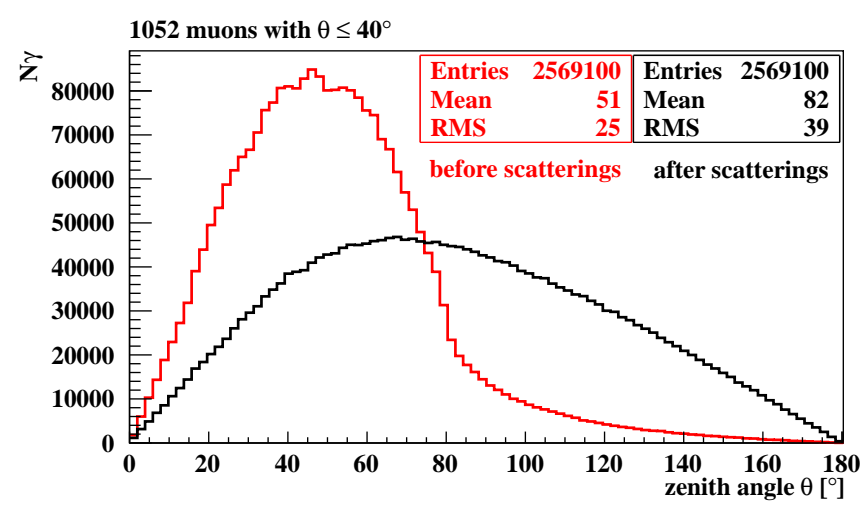

Figure 8: Distribution of zenith angles of the photons that arrive on the surface of the DOMs. The red curve shows the angles at emission. The black curve shows the angles after photons travel in the ice and scatter. The results are the sum of 1052 down-going muons.

After multiple scatterings, the number of photons that are down-going are still higher than those up-going. The ratio is $\sim 1.5: 1$. We then select a set of through-going muon tracks, which are the majority of the background. Events penetrating the detector instrumentation volume by more than $950 \mathrm{~m}$ are included. The photon profile is shown in Figure 9 (left).

The total number of photoelectrons of D-Egg is more than 3 times the NPE value of the IceCube DOM for down-going muons (Figure 9 right). This shows the potential of decreasing the veto volume to gain more effective area for the neutrino searches. 

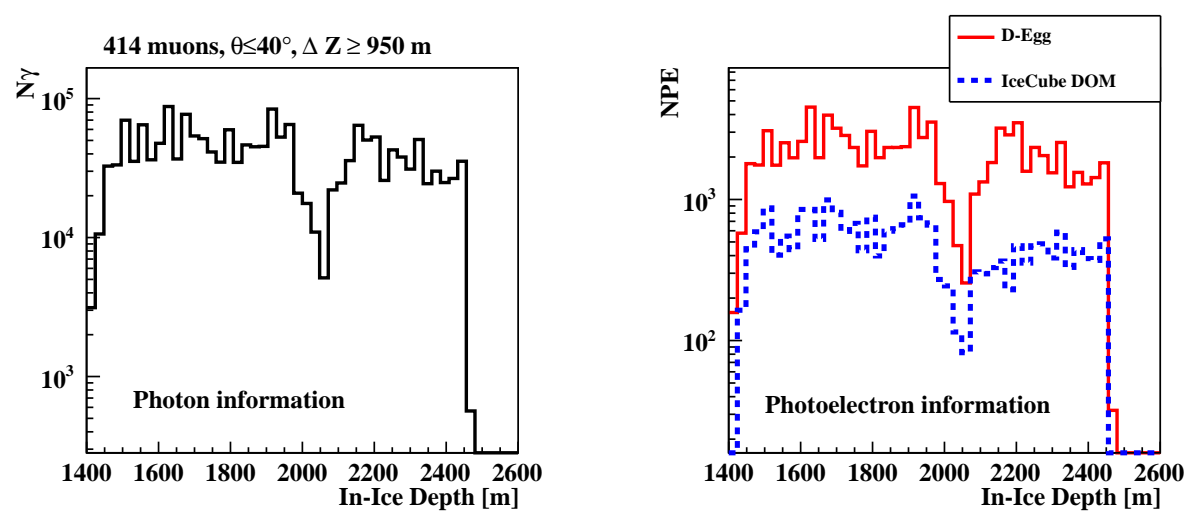

Figure 9: Left: Photon profile for the 414 down-going muons that went through the detector volume vertically. Only photons that arrive on the DOMs are counted. Right: Photoelectron profile of the 414 events detected by D-Egg and IceCube DOM. The dip in the middle is due to dust layer of the ice where the absorption length of photons are small.

\section{Summary}

The D-Egg has been introduced. A DOM with more UV-transparent glass and two PMTs improves the angular sensitivity and the acceptance of Cherenkov photons. By using signal and timing information from two PMTs, we expect it will be possible to reconstruct the event zenith angle and energy with smaller uncertainty and also to increase the effective area of down-going neutrino search.

\section{Acknowledgments}

The authors would like to thank Satoshi Shimizu from Nippon Marine Enterprises, for his great input on the glass design and mechanical tests.

\section{References}

[1] M. G. Aartsen et al. (IceCube-Gen2 Collaboration), IceCube-Gen2: A Vision for the Future of Neutrino Astronomy in Antarctica, (2014)

[2] M. G. Aartsen et al. (IceCube-PINGU Collaboration), Letter of Intent: The Precision IceCube Next Generation Upgrade (PINGU) (2014)

[3] S. Agostinelli et al. (GEANT4 Collaboration), Nucl. Instrum. \& Meth. A506 250 (2003)

[4] R. Abbasi et al. (IceCube Collaboration), Nucl. Instrum. \& Meth. A618 139 (2010)

[5] M. G. Aartsen et al. (IceCube-Gen2 Collaboration), Generation 2 IceCube Digital Optical Module and DAQ, these proceedings, PoS(ICRC2015)1148 (2015)

[6] Hamamatsu Photonics K.K., Photomultiplier Tubes: Basics and Applications, third edition (2006)

[7] M. G. Aartsen et al. (IceCube Collaboration), Astropar. Phys. 3510 (2012)

[8] M. G. Aartsen et al. (IceCube Collaboration), Science 3421242856 (2013)

[9] M. G. Aartsen et al. (IceCube Collaboration), Nucl. Instrum. \& Meth. A711 73 (2013) 